\title{
Resveratrol amplifies BMP-4-stimulated osteoprotegerin synthesis via p38 MAP kinase in osteoblasts
}

\author{
GEN KUROYANAGI ${ }^{1,2}$, HARUHIKO TOKUDA ${ }^{2,3}$, NAOHIRO YAMAMOTO ${ }^{1,2}$, \\ RIE MATSUSHIMA-NISHIWAKI ${ }^{2}$, JUN MIZUTANI ${ }^{1}$, OSAMU KOZAWA ${ }^{2}$ and TAKANOBU OTSUKA ${ }^{1}$ \\ ${ }^{1}$ Department of Orthopedic Surgery, Nagoya City University Graduate School of Medical Sciences, Nagoya, Aichi 467-8601; \\ ${ }^{2}$ Department of Pharmacology, Gifu University Graduate School of Medicine, Gifu 501-1194; \\ ${ }^{3}$ Department of Clinical Laboratory, National Center for Geriatrics and Gerontology, Obu, Aichi 474-8511, Japan
}

Received July 14, 2014; Accepted May 8, 2015

DOI: $10.3892 / \mathrm{mmr} .2015 .3877$

\begin{abstract}
Resveratrol is a naturally occurring polyphenol that possesses health-related properties, and is predominantly found in grapes and berries. Bone morphogenetic protein-4 (BMP-4) stimulates osteocalcin synthesis via p38 mitogen-activated protein (MAP) kinase in osteoblast-like MC3T3-E1 cells. The present study aimed to investigate the effects of resveratrol on BMP-4-induced osteoprotegerin (OPG) synthesis in MC3T3-E1 cells. Resveratrol alone had no effect on OPG expression levels, but significantly enhanced BMP-4-induced OPG release. In addition, resveratrol markedly amplified the mRNA expression levels of BMP-4-induced OPG. SB203580 is an inhibitor of p38 MAP kinase, which was shown to suppress BMP-4-stimulated OPG release. BMP-4-induced phosphorylation of p38 MAP kinase was also enhanced by resveratrol. Furthermore, SB203580 significantly reduced the resveratrol-induced amplification of BMP-4-stimulated OPG release. These results suggested that resveratrol was able to upregulate BMP-4-stimulated OPG synthesis via the amplification of p38 MAP kinase activity in osteoblasts.
\end{abstract}

\section{Introduction}

Bone metabolism is predominantly regulated by two types of functional cells: Osteoblasts and osteoclasts, which are responsible for bone formation and resorption, respectively (1). The formation and remodeling of bone structures result from the combined action of osteoblasts and osteoclasts. Bone resorption and formation are highly regulated in order to maintain adequate bone mass. Osteoblasts also have a role in the regulation of bone resorption via receptor activator

Correspondence to: Dr Haruhiko Tokuda, Department of Clinical Laboratory, National Center for Geriatrics and Gerontology, 7-430 Morioka-cho, Obu, Aichi 474-8511, Japan

E-mail: tokuda@ncgg.go.jp

Key words: resveratrol, osteoblast, bone morphogenetic protein-4, osteoprotegerin of nuclear factor- $\kappa \mathrm{B}$ (RANK) ligand (RANKL) expression, in response to bone resorptive stimuli (2). The binding of RANKL to RANK, which is located at the cell surface of osteoclastic precursors and mature osteoclasts, stimulates osteoclastic differentiation and activation (2). Metabolic bone diseases such as osteoporosis are caused by disordered bone remodeling. Numerous humoral factors are crucial to the bone remodeling process, including prostaglandins such as prostaglandin E2, and cytokines such as interleukin-1 (3).

Osteoprotegerin (OPG) is a secreted protein synthesized by osteoblasts, which inhibits osteoclastic differentiation and activation (4). OPG and RANK belong to the tumor necrosis factor receptor family. OPG acts as a decoy receptor by binding RANKL, which prevents it from binding to RANK, resulting in the suppression of bone resorption (4). RANKL knock-out mice have been shown to suffer from severe osteopetrosis (5). The RANK/RANKL/OPG axis is currently recognized as a major regulatory system for functional osteoclast formation (6).

Bone morphogenetic proteins (BMPs), including transforming growth factor- $\beta$ (TGF- $\beta$ ) and activin, are multifunctional cytokines that belong to the TGF- $\beta$ superfamily (7). BMPs promote bone formation via stimulation of osteoblastic proliferation and differentiation (8). BMP intracellular signaling occurs predominantly via the Smad (Smad 1/5/8)-dependent signaling pathway (5). In addition, previous studies have suggested that Smad-independent signaling, such as mitogen-activated protein (MAP) kinase signaling, also participates in BMP signaling $(9,10)$. A previous study demonstrated that BMP-4 stimulates osteocalcin synthesis in osteoblast-like MC3T3-E1 cells, and that osteocalcin synthesis is positively regulated by p38 MAP kinase (11). In addition, BMP-4 was shown to stimulate the release of vascular endothelial growth factor (VEGF) via p38 MAP kinase in MC3T3-E1 cells (12). BMP also stimulates OPG production in osteoblasts (13). However, the precise mechanism underlying BMP-4-induced OPG synthesis in osteoblasts remains to be elucidated.

It is widely accepted that polyphenolic compounds in foods such as fruits and vegetables are beneficial to humans. Among them, flavonoids exhibit antioxidative, anti-inflammatory and anti-carcinogenic effects $(14,15)$. Resveratrol is a 
naturally occurring polyphenolic flavonoid present in grapes and berries, which has been shown to increase murine life span (16). In addition, the observed low mortality rates due to coronary heart disease in France may be associated with the consumption of wine, which is known to contain abundant resveratrol (17). The effects of resveratrol are mediated by the longevity gene SIRT1, which improves the functioning of cells and organs by activating the $\mathrm{NAD}^{+}$-dependent histone deacetylase (16). NAD ${ }^{+}$is a coenzyme of oxidoreductase synthesized as a precursor of nicotinamide, which has an important role in energy acquisition. Regarding the effects of resveratrol on osteoblasts, a previous study reported that resveratrol was able to stimulate osteoblastic differentiation (18). However, the precise mechanism underlying the effects of resveratrol on bone metabolism remains to be elucidated.

The present study aimed to investigate the effects of resveratrol on BMP-4-stimulated OPG synthesis in osteoblast-like MC3T3-E1 cells.

\section{Materials and methods}

Materials. Resveratrol and SB203580 were purchased from EMD Millipore (Billerica, MA, USA). BMP-4 and mouse OPG ELISA kits were purchased from R\&D Systems, Inc. (Minneapolis, MN, USA). Phospho specific p38 MAP kinase (cat. no. 4511S), p38 MAP kinase antibodies (cat. no. 9212) and anti-rabbit $\mathrm{IgG}$, horseradish peroxidase (HRP)-linked antibodies (cat. no. 7074) were obtained from Cell Signaling Technology (Danvers, MA, USA). The Enhanced Chemiluminescence (ECL) Western Blotting Detection system was obtained from GE Healthcare Life Sciences (Chalfont, UK). $\alpha$-Minimum Essential medium ( $\alpha$-MEM) was obtained from Sigma-Aldrich (St. Louis, MO, USA). Fetal bovine serum (FBS) was obtained from Gibco Life Technologies (Grand Island, NY, USA). Polyvinylidene fluoride membranes were obtained from Bio-Rad Laboratories, Inc. (Berkeley, CA, USA). Resveratrol and SB203580 were dissolved in dimethyl sulfoxide. The maximum concentration of dimethyl sulfoxide was $0.1 \%$, which did not affect the OPG assay or western blot analysis.

Cell culture. Cloned osteoblast like MC3T3 E1 cells were generously provided by Dr M. Kumegawa (Meikai University, Sakado, Japan). The cells were derived from newborn mouse calvaria (19) and were maintained as previously described (20). Briefly, the cells were cultured in $\alpha$-MEM supplemented with $10 \% \mathrm{FBS}$ at $37^{\circ} \mathrm{C}$ in a humidified atmosphere containing $5 \% \mathrm{CO}_{2}$. The cells were subsequently seeded at a density of $5 \times 10^{4}$ cells/dish onto $35 \mathrm{~mm}$ diameter dishes, or at a density of $2 \times 10^{5}$ cells/dish onto $90 \mathrm{~mm}$ diameter dishes in $\alpha$-MEM supplemented with $10 \%$ FBS. Following a period of five days, the medium was exchanged for $\alpha$-MEM supplemented with $0.3 \% \mathrm{FBS}$. The cells were incubated for $48 \mathrm{~h}$ prior to further experimentation.

$O P G$ assay. The cultured cells were treated with $10,30,50$ or $70 \mu \mathrm{M}$ resveratrol or 10,20 or $30 \mu \mathrm{M} \mathrm{SB} 203580$ for $60 \mathrm{~min}$, prior to being stimulated with either $70 \mathrm{ng} / \mathrm{ml}$ of BMP-4 or vehicle in $1 \mathrm{ml} \alpha$-MEM supplemented with $0.3 \% \mathrm{FBS}$, and incubated for $48 \mathrm{~h}$. The vehicle was a solvent, containing phosphate-buffered saline (PBS) supplemented with $0.1 \%$ bovine serum albumin, in which BMP-4 was dissolved. The conditioned medium was subsequently collected, and the OPG concentration was measured using the mouse OPG ELISA kit, according to the manufacturer's instructions. The absorbance was determined using an ELISA plate reader (Multiskan JX; Thermo Labsystems, Inc., Franklin, MA, USA).

Western blot analysis. The cultured cells were treated with 10,30 or $50 \mu \mathrm{M}$ resveratrol for $60 \mathrm{~min}$, prior to being stimulated with either $70 \mathrm{ng} / \mathrm{ml} \mathrm{BMP-4}$ or vehicle in $1 \mathrm{ml} \alpha$-MEM supplemented with $0.3 \%$ FBS for $2 \mathrm{~h}$. The cells were then washed twice with PBS prior to being lysed. Adherent cells were scraped off the dish using a plastic cell scraper, then the cell suspension was gently transferred into a microcentrifuge tube in a lysis buffer containing $62.5 \mathrm{mM}$ Tris/ $\mathrm{HCl}$ (pH 6.8), $2 \%$ SDS, $50 \mathrm{mM}$ dithiothreitol, and $10 \%$ glycerol, and then sonicated with 20 short burst of $1 \mathrm{sec}$. SDS-PAGE was performed according to the Laemmli method (21) in $10 \%$ polyacrylamide gels. A western blot analysis was performed as previously described (22). The membranes were blocked with 5\% fat-free dry milk in Tris-buffered saline with Tween (TBST; $20 \mathrm{mM}$ Tris/ $\mathrm{HCl}, \mathrm{pH} 7.6,137 \mathrm{mM} \mathrm{NaCl}, 0.1 \%$ Tween-20) for $1 \mathrm{~h}$ prior to incubation with the primary antibodies. The membranes were then incubated with the primary antibodies targeting phospho specific p38 MAP kinase and p38 MAP kinase, all at a dilution of 1:1,000 in 5\% milk in TBST overnight at $4^{\circ} \mathrm{C}$, using goat peroxidase-labeled antibodies and anti-rabbit IgG HRP-linked secondary antibody (cat. no. 7074) at a dilution of 1:1,000 in 5\% milk in TBST for $1 \mathrm{~h}$ at room temperature (Cell Signaling Technology). The peroxidase activity on the polyvinylidene fluoride membrane (Bio-Rad Laboratories, Inc.) was visualized on an X-ray film using the ECL Western Blotting Detection system.

Reverse transcription-quantitative polymerase chain reaction $(R T-q P C R)$. The cultured cells were treated with either $50 \mu \mathrm{M}$ resveratrol or vehicle for $60 \mathrm{~min}$, prior to being stimulated with either $70 \mathrm{ng} / \mathrm{ml} \mathrm{BMP-4}$ or vehicle in $\alpha$-MEM supplemented with $0.3 \%$ FBS for $3 \mathrm{~h}$. Total RNA was isolated and reverse transcribed into cDNA using TRIzol ${ }^{\circledR}$ reagent (Invitrogen Life Technologies, Carlsbad, CA, USA) and Omniscript Reverse Transcriptase kit (Qiagen Inc., Valencia, CA, USA). RT-qPCR was performed in capillaries using a Light Cycler 1.2 system and the Fast Start DNA Master SYBR Green I kit (Roche Diagnostics, Basel, Switzerland). The sense and antisense primers (primer set ID: MA026526) for mouse OPG and GAPDH mRNA were purchased from Takara Bio, Inc. (Otsu, Japan). The primer sequences were as follows: Forward 5'-CAATGG CTGGCTTGGTTTCATAG-3' and reverse 5'-CTGAACCAG ACATGACAGCTGGA-3'. The sense and antisense primers for mouse GAPDH mRNA were synthesized based on the study of Simpson et al (23) and obtained from Sigma-Aldrich. The primer sequences were as follows: Forward 5'-AACGACCCCTTCATT GAC-3' and reverse 5'-TCCACGACATACTCAGCAC-3'. The reaction mixtures were incubated at $95^{\circ} \mathrm{C}$ for $10 \mathrm{~min}$, followed by 40 cycles at $60^{\circ} \mathrm{C}$ for $5 \mathrm{sec}$ and $72^{\circ} \mathrm{C}$ for $7 \mathrm{sec}$. The amplified products were determined by melting curve analysis and $1 \%$ agarose gel electrophoresis. The mRNA expression levels of OPG were normalized to those of GAPDH. 
Densitometric analysis. A densitometric analysis was performed using ImageJ 1.32 (National Institutes of Health, Bethesda, MA, USA) image analysis software program. The background-subtracted signal intensity of each phosphorylation signal was normalized to the respective total protein signal and plotted as the fold increase, as compared with non-stimulated control cells.

Statistical analysis. Differences between the mean values for individual groups were assessed with a one-way analysis of variance, followed by a Bonferroni analysis in order to carry out multiple comparisons between pairs. Microsoft Excel 2010 (Redmond, WA, USA) was used for statistical analysis. $\mathrm{P}<0.05$ was considered to indicate a statistically significant difference. All data are presented as the mean \pm standard error of the mean, and all experiments were performed in triplicate from three independent cell preparations.

\section{Results}

Effects of resveratrol on BMP-4-stimulated OPG release in MC3T3-E1 cells. BMP-4 has previously been shown to stimulate the synthesis of osteocalcin and VEGF in osteoblast-like MC3T3-E1 cells $(11,12)$. In addition, BMP-2 induces the synthesis of OPG in human osteoblasts (13). The present study investigated whether BMP-4 stimulated OPG synthesis in MC3T3-E1 cells. The results indicated that BMP-4 significantly increased the release of OPG (Fig. 1). The present study also investigated the effects of resveratrol on BMP-4-stimulated OPG release. The results suggested that although resveratrol alone had little effect on the synthesis of OPG, it significantly enhanced BMP-4-stimulated OPG release in a dose-dependent manner $(10-70 \mu \mathrm{M})$ in MC3T3-E1 cells (Fig. 1). The maximum effect of resveratrol was observed at $70 \mu \mathrm{M}$, which caused a $\sim 230 \%$ increase in the release of OPG.

Effects of resveratrol on the BMP-4-induced $m R N A$ expression of $O P G$ in MC3T3-El cells. The results of the RT-qPCR demonstrated that BMP-4 induced the mRNA expression of OPG in osteoblast-like MC3T3-E1 cells (Fig. 2). In order to investigate whether the amplifying effects of resveratrol on BMP-4-stimulated OPG release were mediated through transcription, the effects of resveratrol on BMP-4-induced OPG mRNA expression were also examined. Resveratrol alone failed to affect the mRNA expression levels of OPG, but markedly enhanced BMP-4-induced OPG mRNA expression (Fig. 2). These findings were concordant with the observed increase in BMP-4-stimulated OPG release following resveratrol treatment.

Effects of SB203580 on BMP-4-stimulated OPG release in MC3T3-E1 cells. Results from previous studies have suggested that Smad-independent signaling, such as MAP kinase signaling, mediates the effects of BMPs $(9,10)$. p38 MAP kinase has previously been reported to function in BMP-4-stimulated osteocalcin synthesis as a positive regulator in osteoblast-like MC3T3-E1 cells (11). p38 MAP kinase has also been shown to upregulate BMP-4-stimulated VEGF synthesis in MC3T3-E1 cells (12). In order to determine whether p38 MAP kinase is involved in BMP-4-induced OPG synthesis in MC3T3-E1 cells,

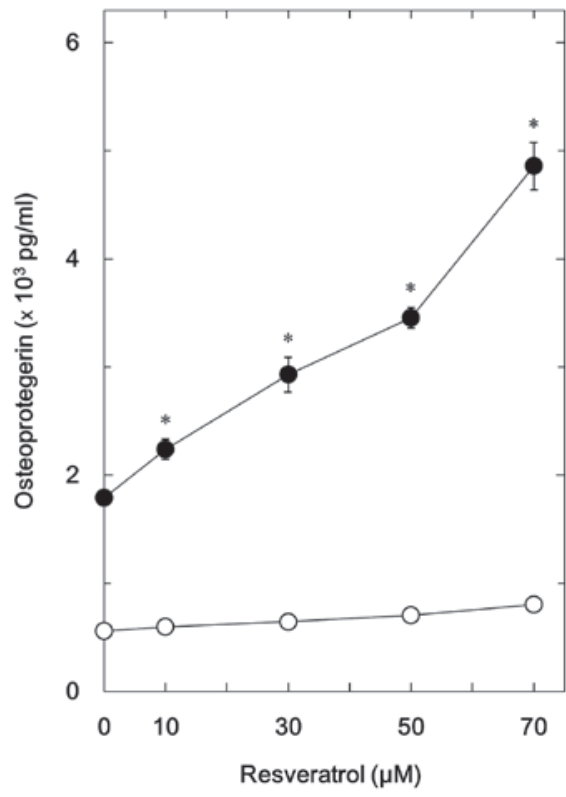

Figure 1. Effects of resveratrol on bone morphogenetic protein (BMP)-4-stimulated osteoprotegerin (OPG) release in MC3T3-E1 osteoblastic-like cells. The cultured cells were treated with various doses of resveratrol for $60 \mathrm{~min}$, prior to being stimulated with either $70 \mathrm{ng} / \mathrm{ml}$ BMP-4 (black) or vehicle (white) for $48 \mathrm{~h}$. The OPG concentrations of the culture medium were determined by ELISA. Each value is presented as the mean \pm standard error of the mean. The experiments were performed in triplicate from three independent cell preparations. ${ }^{*} \mathrm{P}<0.05$, vs. treatment with BMP-4 alone.

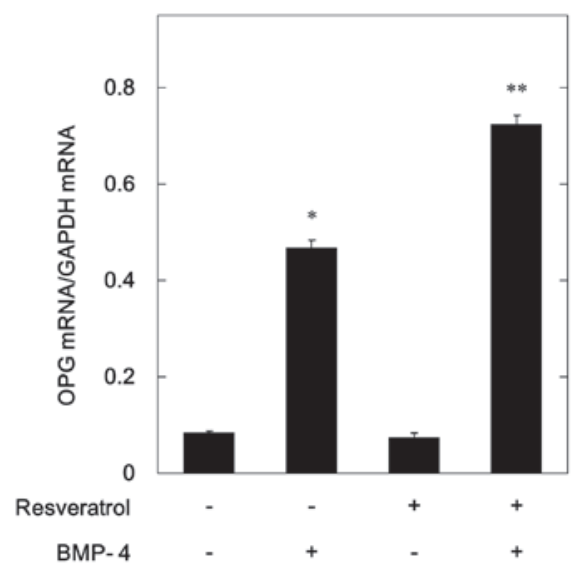

Figure 2. Effects of resveratrol on bone morphogenetic protein (BMP)-4-induced osteoprotegerin (OPG) mRNA expression in MC3T3-E1 osteoblastic-like cells. The cultured cells were treated with either $50 \mu \mathrm{M}$ resveratrol or vehicle for $60 \mathrm{~min}$, prior to being stimulated with either $70 \mathrm{ng} / \mathrm{ml} \mathrm{BMP}-4$ or vehicle for $3 \mathrm{~h}$. The total RNA was then isolated and reverse transcribed into cDNA. The mRNA expression levels of OPG and GAPDH were quantified by reverse trancription-quantitative polymerase chain reaction. The mRNA expression levels of OPG were normalized to those of GAPDH. Each value is presented as the mean \pm standard error of the mean. The experiments were performed in triplicate from three independent cell preparations. ${ }^{*} \mathrm{P}<0.05$, vs. the control. ${ }^{* *} \mathrm{P}<0.05$, vs. treatment with BMP-4 alone.

the effects of SB203580, an inhibitor of p38 MAP kinase (24), were examined on BMP-4-stimulated OPG release. Treatment with SB203580 alone had little effect on OPG release, but significantly reduced BMP-4-stimulated OPG release in a dose-dependent manner (10-30 $\mu \mathrm{M})$ (Fig. 3). 
Table I. Effects of SB203580 on the resveratrol enhancement of BMP-4-stimulated OPG release in MC3T3-E1 osteoblastic-like cells.

\begin{tabular}{lccc}
\hline SB203580 & Resveratrol & BMP-4 & OPG $(\mathrm{pg} / \mathrm{ml})$ \\
\hline- & - & - & $576 \pm 17$ \\
- & - & + & $2,268 \pm 46^{\mathrm{a}}$ \\
- & + & - & $632 \pm 14$ \\
- & + & + & $4,610 \pm 265^{\mathrm{b}}$ \\
+ & - & + & $443 \pm 31$ \\
+ & - & - & $948 \pm 16^{\mathrm{b}}$ \\
+ & + & - & $457 \pm 20$ \\
+ & + & + & $1,522 \pm 41^{\mathrm{c}}$ \\
\hline
\end{tabular}

The cultured cells were treated with either $10 \mu \mathrm{M} \mathrm{SB} 203580$ or vehicle for $60 \mathrm{~min}$, prior to being incubated with either $50 \mu \mathrm{M}$ resveratrol or vehicle for $60 \mathrm{~min}$. The cells were subsequently stimulated with either $50 \mathrm{ng} / \mathrm{ml}$ bone morphogenetic protein (BMP)-4 or vehicle for $48 \mathrm{~h}$. The osteoprotegerin (OPG) concentrations in the culture medium were determined by ELISA. Each value is presented as the mean \pm standard error of the mean. The experiments were performed in triplicate from three independent cell preparations. ${ }^{a} \mathrm{P}<0.05$, vs. the control. ${ }^{b} \mathrm{P}<0.05$, vs treatment with BMP-4 alone. ${ }^{c} \mathrm{P}<0.05$, vs. treatment with BMP-4 and resveratrol.

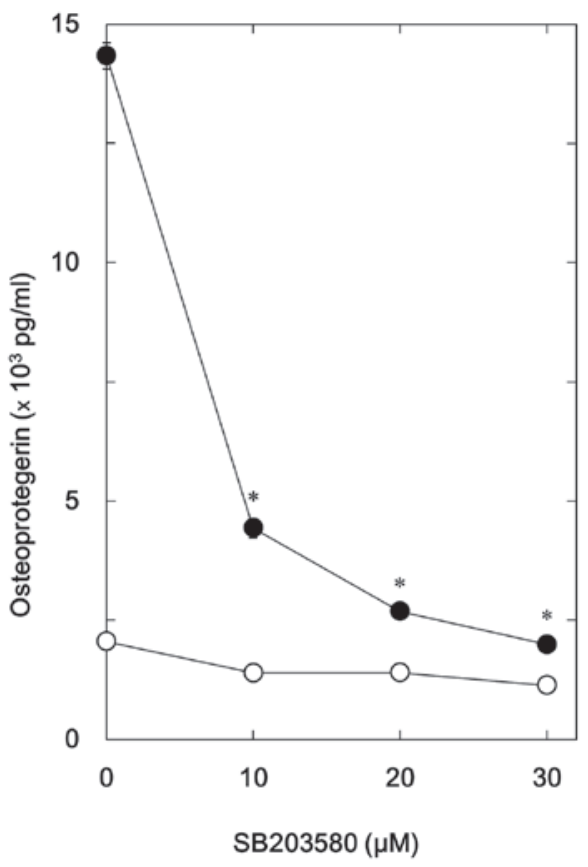

Figure 3. Effects of SB203580 on bone morphogenetic protein (BMP)-4-stimulated osteoprotegerin (OPG) release in MC3T3-E1 osteoblastic-like cells. The cultured cells were treated with various doses of SB203580 for $60 \mathrm{~min}$, prior to being stimulated with either $70 \mathrm{ng} / \mathrm{ml}$ BMP-4 (black) or vehicle (white) for $48 \mathrm{~h}$. The OPG concentrations in the culture medium were determined by ELISA. Each value is presented as the mean \pm standard error of the mean. All experiments were performed in triplicate from three independent cell preparations. ${ }^{*} \mathrm{P}<0.05$, vs. treatment with BMP-4 alone.

Effects of resveratrol on BMP-4-induced phosphorylation of p38 MAP kinase in MC3T3-E1 cells. In order to clarify whether the enhancing effects of resveratrol on BMP-4-stimulated OPG synthesis were mediated by the modulation of $\mathrm{p} 38$ MAP kinase activation in osteoblast-like MC3T3-E1 cells, the effects of resveratrol on BMP-4-induced phosphorylation of p38 MAP kinase were examined. The results indicated that resveratrol significantly enhanced BMP-4-induced phosphorylation of p38

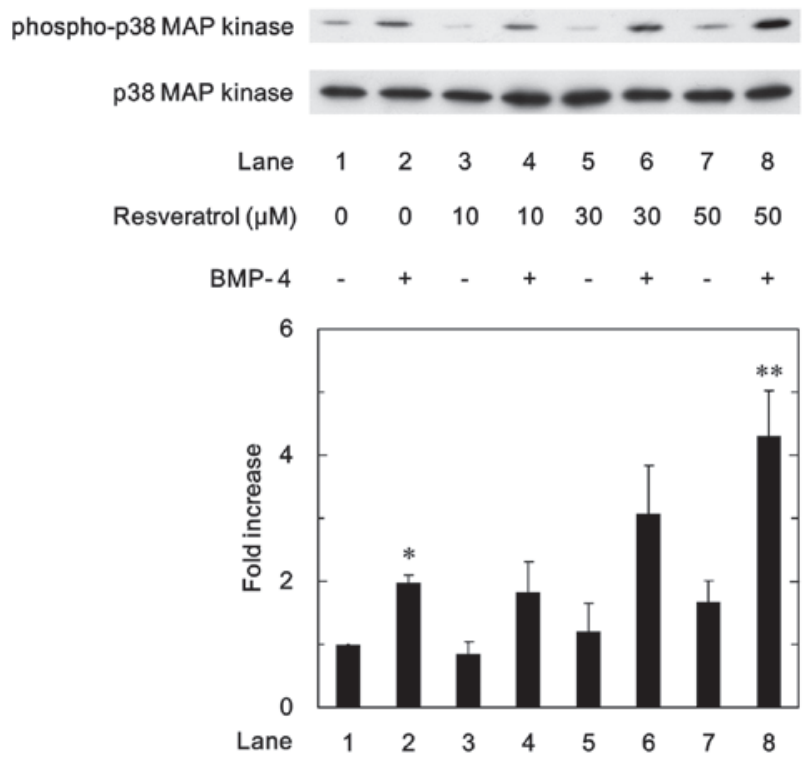

Figure 4. Effects of resveratrol on the bone morphogenetic protein (BMP)-4-induced phosphorylation of $\mathrm{p} 38$ mitogen-activated protein (MAP) kinase in MC3T3-E1 osteoblast-like cells. The cultured cells were treated with various doses of resveratrol for $60 \mathrm{~min}$, prior to being stimulated with either $70 \mathrm{ng} / \mathrm{ml} \mathrm{BMP}-4$ or vehicle for $2 \mathrm{~h}$. The cell extracts were then subjected to SDS-PAGE and western blotting, using antibodies targeting phospho-specific p38 MAP kinase or p38 MAP kinase. The histogram shows the quantitative representation of the levels of BMP-4-induced phosphorylation obtained from densitometric analyses. Each value is presented as the mean \pm standard error of the mean. All experiments were performed in triplicate from three independent cell preparations. ${ }^{*} \mathrm{P}<0.05$, vs. the control. ${ }^{* *} \mathrm{P}<0.05$, vs. treatment with $\mathrm{BMP}-4$ alone.

MAP kinase in a dose-dependent manner (10-50 $\mu \mathrm{M})$ (Fig. 4). Conversely, a previous study demonstrated that resveratrol did not affect BMP-4-induced phosphorylation of Smad 1/5/8 in MC3T3-E1 cells (25).

Effects of SB203580 on resveratrol enhancement of BMP-4-stimulated OPG release in MC3T3-E1 cells. The present study examined the effects of SB203580 on 
resveratrol enhancement of BMP-4-stimulated OPG release in MC3T3-E1 cells. The results indicated that SB203580 significantly suppressed the resveratrol-induced amplification of BMP-4-stimulated OPG release (Table I). SB203580 caused a $\sim 70 \%$ decrease in the effects of resveratrol-enhanced BMP-4.

\section{Discussion}

The results of the present study demonstrated that resveratrol significantly enhanced BMP-4-stimulated OPG release in osteoblast-like MC3T3-E1 cells. In addition, resveratrol was able to amplify BMP-4-induced mRNA expression levels of OPG in MC3T3-E1 cells. Therefore, it is likely that the enhancing effects of resveratrol on BMP-4-stimulated OPG release are mediated through transcriptional events. To the best of our knowledge, this is the first study to report the resveratrol enhancement of BMP-4-stimulated OPG synthesis in osteoblasts.

Based on these results, the mechanism underlying resveratrol enhancement of BMP-4-induced OPG synthesis was investigated in the osteoblast-like cells. Previous studies have demonstrated that the effects of BMPs are exerted through the intracellular signaling of both Smad-dependent, and Smad-independent pathways such as MAP kinase signaling $(7,9,10)$. In Smad-dependent signaling, resveratrol previously failed to affect BMP-4-induced phosphorylation of Smad 1/5/8 in osteoblast-like MC3T3-E1 cells (25). These results suggested that it is unlikely that the resveratrol enhancement of BMP-4-induced OPG synthesis is mediated through the Smad-dependent pathway in osteoblast-like MC3T3-E1 cells. Previous studies have demonstrated that p38 MAP kinase acts as a positive regulator in the BMP-4-stimulated synthesis of both osteocalcin and VEGF in osteoblast-like MC3T3-E1 cells $(11,12)$. In addition, a previous study examined the effects of SB203580 on BMP-4-stimulated OPG synthesis in MC3T3-E1 cells, and demonstrated that SB203580 significantly reduced BMP-4-stimulated OPG release (24). The results of the present study suggested that p38 MAP kinase was also involved in BMP-4-stimulated OPG synthesis in osteoblast-like MC3T3-E1 cells. In addition, resveratrol was shown to markedly enhance BMP-4-induced phosphorylation of p38 MAP kinase in MC3T3-E1 cells. Furthermore, SB203580 significantly suppressed the resveratrol enhancement of BMP-4-stimulated OPG release. These results indicated that the resveratrol amplification of BMP-4-stimulated OPG synthesis may be mediated through the upregulation of p38 MAP kinase activity in osteoblast-like MC3T3-E1 cells.

Resveratrol is a naturally occurring polyphenol that is abundantly present in grapes and berries, and exhibits numerous beneficial effects on human health, such as antioxidation, anti-aging, and reduction of stress $(26,27)$. BMP-4 increases bone formation and is an important regulator of fracture healing (8). OPG is a decoy receptor of RANKL, which blocks RANK/RANKL interaction thereby preventing osteoclast differentiation and activation (4). The results of the present study suggested that the resveratrol enhancement of BMP-4-induced OPG synthesis led to an increase in bone formation, and a suppression of bone resorption. Therefore, resveratrol may prove useful in the treatment of skeletal conditions via modulation of osteoblast function. Further investigation is required in order to clarify the mechanism underlying resveratrol amplification of OPG synthesis in osteoblasts.

In conclusion, the results of the present study suggested that resveratrol was able to upregulate BMP-4-stimulated OPG synthesis via amplification of p38 MAP kinase activity in osteoblasts.

\section{Acknowledgements}

The authors of the present study are grateful for the technical assistance of Mrs. Yumiko Kurokawa (Department of Pharmacology, Gifu University Graduate School of Medicine). The present study was supported by grants from the Ministry of Education, Science, Sports and Culture of Japan (grant no. 19591042), the Foundation for Growth Science (grant no. 23-9), and the Ministry of Health, Labour and Welfare of Japan (grant no. 25-4).

\section{References}

1. Karsenty $G$ and Wagner EF: Reaching a genetic and molecular understanding of skeletal development. Dev Cell 2: 389-406, 2002.

2. Boyce BF, Rosenberg E, de Papp AE and Duong le T: The osteoclast, bone remodelling and treatment of metabolic bone disease. Eur J Clin Invest 42: 1332-1341, 2012.

3. Parfitt AM: Targeted and nontargeted bone remodeling: Relationship to basic multicellular unit origination and progression. Bone 30: 5-7, 2002.

4. Simonet WS, Lacey DL, Dunstan CR, Kelley M, Chang MS, Lüthy R, Nguyen HQ, Wooden S, Bennett L and Boone T: Osteoprotegerin: A novel secreted protein involved in the regulation of bone density. Cell 89: 309-319, 1997.

5. Mizuno A, Kanno T, Hoshi M, Shibata O, Yano K, Fujise N, Kinosaki M, Yamaguchi K, Tsuda E, Murakami A, et al: Transgenic mice overexpressing soluble osteoclast differentiation factor (sODF) exhibit severe osteoporosis. J Bone Miner Metab 20: 337-344, 2002.

6. Kwan Tat S, Padrines M, Théoleyre S, Heymann D and Fortun Y: IL-6, RANKL, TNF-alpha/IL-1: Interrelations in bone resorption pathophysiology. Cytokine Growth Factor Rev 15: 49-60, 2004.

7. Miyazono K, Maeda S and Imamura T: BMP receptor signaling: Transcriptional targets, regulation of signals, and signaling cross-talk. Cytokine Growth Factor Rev 16: 251-263, 2005.

8. Krause C, de Gorter DJJ, Karperien M and ten Dijke P: Signal transduction cascades controlling osteoblast differentiation. In: Primer on the Metabolic Bone Diseases and Disorders of Mineral Metabolism. Rosen CJ (ed). 7th edition. ASBMR, Washington, 2008.

9. Moustakas A and Heldin CH: Non-Smad TGF-beta signals. J Cell Sci 118: 3573-3584, 2005.

10. Cai J, Pardali E, Sánchez-Duffhues G and ten Dijke P: BMP signaling in vascular diseases. FEBS Lett 586: 1993-2002, 2012.

11. Kozawa O, Hatakeyama D and Uematsu T: Divergent regulation by p44/p42 MAP kinase and p38 MAP kinase of bone morphogenetic protein-4-stimulated osteocalcin synthesis in osteoblasts. J Cell Biochem 84: 583-589, 2002.

12. Tokuda H, Hatakeyama D, Shibata T, Akamatsu S, Oiso Y and Kozawa O: p38 MAP kinase regulates BMP-4-stimulated VEGF synthesis via p70 S6 kinase in osteoblasts. Am J Physiol Endocrinol Metab 284: E1202-E1209, 2003.

13. Hofbauer LC, Dunstan CR, Spelsberg TC, Riggs BL and Khosla S: Osteoprotegerin production by human osteoblast lineage cells is stimulated by vitamin D, bone morphogenetic protein-2, and cytokines. Biochem Biophys Res Commun 250: 776-781, 1998.

14. Jankun J, Selman SH, Swiercz R and Skrzypczak-Jankun E: Why drinking green tea could prevent cancer. Nature 387: 561, 1997.

15. Harborne JB and Williams CA: Advances in flavonoid research since 1992. Phytochemistry 55: 481-504, 2000. 
16. Baur JA, Pearson KJ, Price NL, Jamieson HA, Lerin C, Kalra A Prabhu VV, Allard JS, Lopez-Lluch G, Lewis K, et al: Resveratrol improves health and survival of mice on a high-calorie diet. Nature 444: 337-342, 2006.

17. Renaud S and de Lorgeril M: Wine, alcohol, platelets, and the French paradox for coronary heart disease. Lancet 339: 1523-1526, 1992.

18. MizutaniK,IkedaK,Kawai Yand Yamori Y:Resveratrolstimulates the proliferation and differentiation of osteoblastic MC3T3-E1 cells. Biochem Biophys Res Commun 253: 859-863, 1998.

19. Sudo H, Kodama HA, Amagai Y, Yamamoto S and Kasai S: In vitro differentiation and calcification in a new clonal osteogenic cell line derived from newborn mouse calvaria. J Cell Biol 96: 191-198, 1983.

20. Kozawa O, Tokuda H, Miwa M, Kotoyori J and Oiso Y: Cross-talk regulation between cyclic AMP production and phosphoinositide hydrolysis induced by prostaglandin E2 in osteoblast-like cells. Exp Cell Res 198: 130-134, 1992.

21. Laemmli UK: Cleavage of structural proteins during the assembly of the head of bacteriophage T4. Nature 227: 680-685, 1970.
22. Kato $\mathrm{K}$, Ito $\mathrm{H}$, Hasegawa $\mathrm{K}$, Inaguma $\mathrm{Y}$, Kozawa $\mathrm{O}$ and Asano T: Modulation of the stress-induced synthesis of hsp27 and alpha B-crystallin by cyclic AMP in C6 rat glioma cells. J Neurochem 66: 946-950, 1996.

23. Simpson DA, Feeney S, Boyle C and Stitt AW: Retinal VEGF mRNA measured by SYBR green I fluorescence: A versatile approach to quantitative PCR. Mol Vis 6: 178-183, 2000.

24. Cuenda A, Rouse J, Doza YN, Meier R, Cohen P, Gallagher TF, Young PR and Lee JC: SB 203580 is a specific inhibitor of a MAP kinase homologue which is stimulated by cellular stresses and interleukin-1. FEBS Lett 364: 229-233, 1995.

25. Kondo A, Otsuka T, Kuroyanagi G, Yamamoto N, Matsushima-Nishiwaki R, Mizutani J, Kozawa $\mathrm{O}$ and Tokuda $\mathrm{H}$ : Resveratrol inhibits BMP-4-stimulated VEGF synthesis in osteoblasts: Suppression of S6 kinase. Int J Mol Med 33: 1013-1018, 2014.

26. Blander G and Guarente L: The Sir2 family of protein deacetylases. Annu Rev Biochem 73: 417-435, 2004.

27. Koo SH and Montminy M: In vino veritas: a tale of two sirtls? Cell 127: 1091-1093, 2006. 